\title{
Correction to: FMNL1 mediates nasopharyngeal carcinoma cell aggressiveness by epigenetically upregulating MTA1
}

Wen-Hui Chen · Mu-Yan Cai · Jia-Xing Zhang • Feng-Wei Wang • Lin-Quan Tang • Yi-Ji Liao • Xiao-Han Jin • Chen-Yuan Wang • Ling Guo · Yi-Guo Jiang • Cai-Ping Ren • Hai-Qiang Mai • Mu-Sheng Zeng (D) Hsiang-Fu Kung • Chao-Nan Qian · Dan Xie

Published online: 25 March 2021

(c) The Author(s), under exclusive licence to Springer Nature Limited 2021

\section{Correction to: Oncogene}

https://doi.org/10.1038/s41388-018-0351-8

Published online 16 July 2018

Following the publication of the above article, the authors noted an error in Fig. 3g (down, right), in which it should be from lung metastatic tumors of mice that originated
S26-FMNL1-shMTA1 cells. However, we found that this image was duplicated and misused from S18-shFMNL1 cells in Fig. 2g (down, right). We apologize for any inconvenience caused by this careless error.

This correction notice contains the original image from the lung metastatic tumors originated S26-FMNL1shMTA1 cells, and was completely corrected in Fig. 3g. 
a

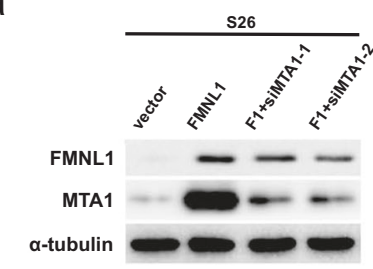

b

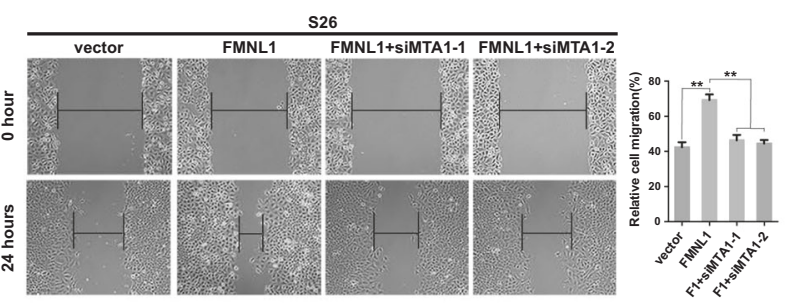

C

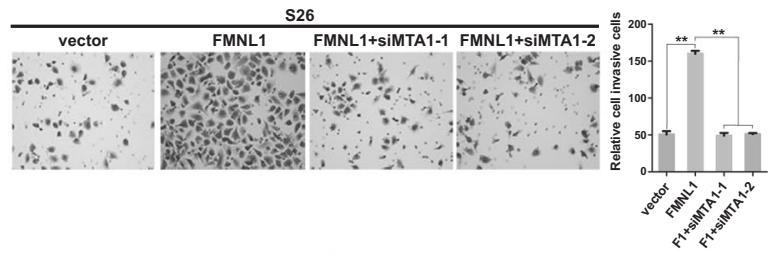

e

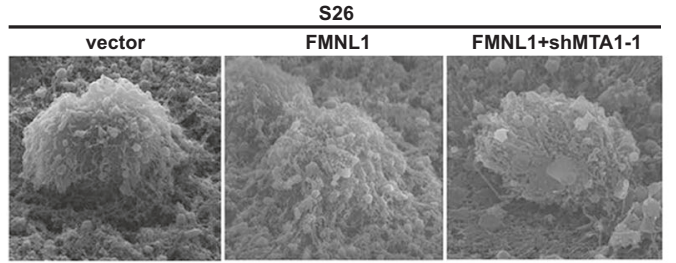

f
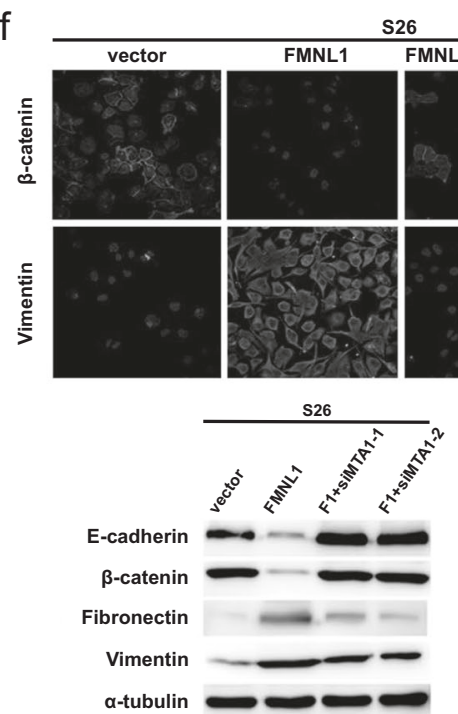

d
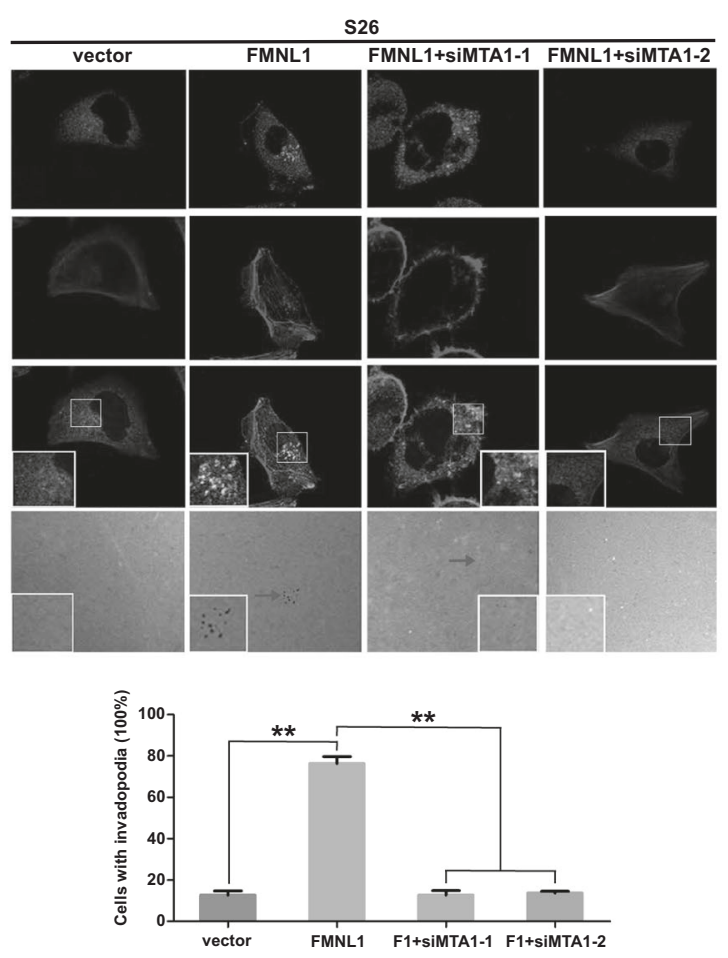

g
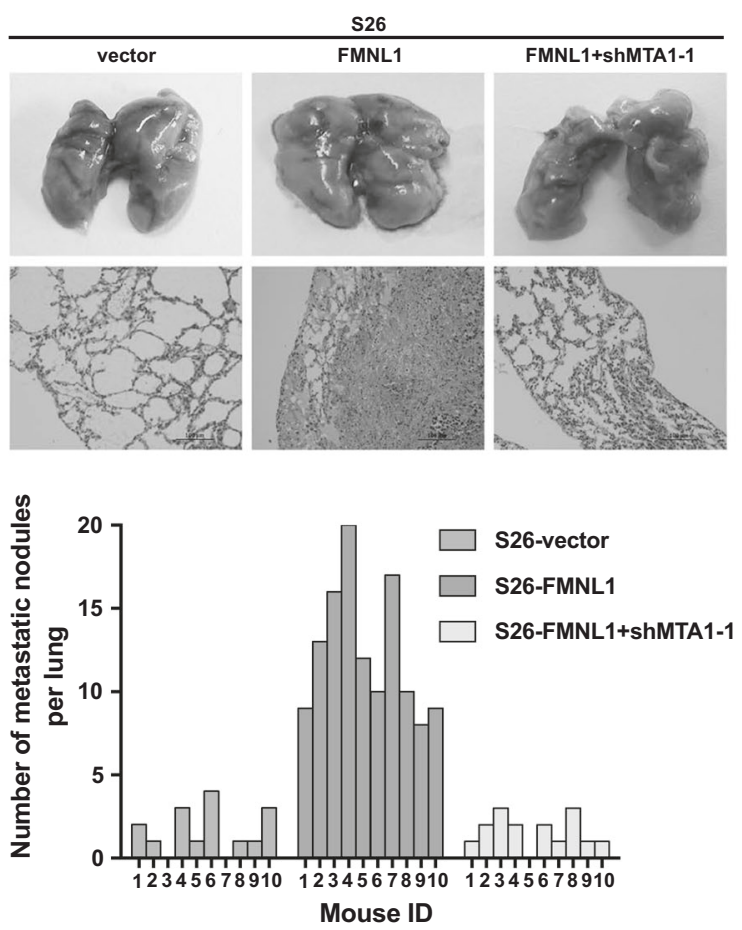
Fig. 3 MTA1 is involved in FMNL1-induced NPC invasiveness, invadopodia formation, and EMT in vitro and metastasis in vivo. a Treatment of two siMTA1s in S26-FMNL1 and SUNE2-FMNL1 cells efficiently decreased the expression levels of MTA1 as detected by western blotting. b Silencing of MTA1 by siMTA1 in S26FMNL1cells significantly inhibited cell migration ability as examined by wound-healing assays. **, $P<0.01$. c Silencing of MTA1 substantially decreased the invasion of S26-FMNL1 cells, analyzed by transwell assays. **, $P<0.01$. d IF staining shows that S26-FMNL1 cells had more invadopodia formation (coexpression of F-actin and cortactin) compared with control 26-vector cells. Knockdown of MTA1 in S26-FMNL1 cells dramatically inhibited cell invadopodia formation. F-actin was stained red, whereas cortactin green and nuclei blue. $* *, P<0.01$. e Scanning electron microscope examination demonstrates S26-FMNL1 more progressively invaded the matrix after $12 \mathrm{~h}$ compared with S26-vector cells, whereas depletion of MTA1 in S26-FMNL1 cells inhibited the adherence effect of invadopodia. Filopodia-like protrusions and some membrane blebs were observed between the free surface of invading cells and matrigel. f Western blotting shows that after silence of MTA1 in S26-FMNL1 cells, the levels of E-cadherin and $\beta$-catenin increased, whereas fibronectin and Vimentin levels decreased (bottom). IF staining demonstrating a downregulated expression of $\beta$-catenin and an upregulated expression of Vimentin in S26-FMNL1 cells after depletion of MTA1 (upper). g Silencing of MTA1 by shMTA1 substantially inhibited FMNL1-induced S26 cell pulmonary metastasis in nude mice 6 weeks after tail vein injection of cells. Ten mice per group; $P<0.01$. 